\title{
Effects of Artemisia dracunculus Aqueous Extract on Blood Sugar, Serum Insulin, Triglyceride and Liver Enzymes in Fructose Drinking Water Male Rats
}

\author{
Mohammad Reza Shahraki, ${ }^{1,}$ Hamideh Mirshekari, ${ }^{2}$ Zahra Samadi, ${ }^{3}$ Ahmad Reza Shahraki, ${ }^{4}$ and \\ Elham Shahraki ${ }^{5}$ \\ ${ }^{1}$ Department of Physiology, Faculty of Medicine, Zahedan University of Medical Sciences and Health Services, Zahedan, Iran \\ ${ }^{2}$ Zahedan Health Center, Zahedan University of Medical Sciences, Zahedan, Iran \\ ${ }^{3}$ Department of Physiology, Zahedan University of Medical Sciences and Health Services, Zahedan, Iran \\ ${ }^{4}$ Zahedan University of Medical Sciences and Health Services, Zahedan, Iran \\ ${ }^{5}$ Imam Ali Hospital, Zahedan University of Medical Sciences and Health Services, Zahedan, Iran \\ "Corresponding author: Mohammad Reza Shahraki, Department of Physiology, Faculty of Medicine, Zahedan University of Medical Sciences and Health Services, Zahedan, Iran. \\ E-mail: iranmirshekari@yahoo.com
}

Received 2015 October 18; Revised 2016 July 27; Accepted 2017 February 08.

\begin{abstract}
Background: Artemisia are various groups of plants which are used as an herbal medicine in all countries; the present study was designed to evaluate the effects of Artemisia dracunculus (AD) leaves aqueous extract on blood sugar, serum insulin, and triglyceride and liver enzymes in Fructose Drinking water (FDW) male rats.

Methods: At the beginning of experiment, 48 Wistar-albino male rats, weighing $200-250$ g were divided into control (C) and FDW groups $(n=24)$. FDW group received $\mathrm{FDW}(10 \%, \mathrm{w} / \mathrm{v})$ for a month but control group did not receive any agents during the trial period. A half of control and FDW groups received AD L aqueous extract daily during trial period. At the end, animals were anesthetized, sacrificed and blood samples were collected from cervical vessels. Serum insulin, Blood glucose, insulin resistance index, triglyceride and liver enzymes were measured by ordinary methods. Obtained data were analyzed using SPSS-17 via one way ANOVA and Tukey tests.

Results: Our results showed that serum insulin, blood sugar, insulin resistance index, triglyceride, Aspartate amino transferase (AST) and Alanine amino transferase (ALT) values in FDW group significantly increased compared to $\mathrm{C}$ and $\mathrm{C}+\mathrm{E}$ groups but these values in group FDW + E were significantly decreases compared to group FDW $(\mathrm{P}<0.001)$.

Conclusions: Our findings demonstrated that AD L aqueous extract improves blood sugar, serum insulin, insulin resistance index and liver enzymes in rat model.
\end{abstract}

Keywords: Artemisia dracunculuss, Blood Sugar, Insulin Resistance, Insulin, Insulin, Rat

\section{Background}

Unnecessary fructose intake, characteristic for modern diet loaded in polished sugar has been associated with the augmentation of metabolic syndrome [1]. Metabolic syndrome is described the group of character of quite a lot of metabolic abnormalities, based on hyperlipidemia, abdominal obesity and insulin resistance [2]. This disorder is accompanied with an increase in serum insulin, leptin hormone and the possibility for atherosclerotic cardiovascular disease and type 2 diabetes mellitus [3]. Research indicated that in fructose- fed rats (FFR) circulating triglyceride, the weight of liver, abdominal adipose tissue, and insulin resistance (IR) increased that was accompanied with impaired glucose tolerance [4]. Moreover, in FFR metabolic syndrome is induced which is correlated to hyperglycemia, dyslipidemia and insulin resistance [5-
7]. Artemisia dracunculus (AD) is an herbal medicine whose extract has been shown to improve insulin sensitivity in vitro and in vivo studies [8]. The experiment indicated that Artemisinin is a powerful drug which obtained from Artemisia is a possible contraceptive agent and has shown anti malarial activity [9]. Moreover aqueous extract of Artemisia capillaries has shown a hepatoprotective effects on FFR [10]. Artemisia extract affected on insulin sensitivity, modulating of carbohydrate metabolism and glucose influx [11]. Investigation revealed that Artemisia extracts had shown hepatoprotective and hypolipidemic properties and antioxidative activity in hepatoma cells in Dgalactose-induced oxidative stress in mouse model [12-14]. Literature indicate that Artemisia contain an active compound which affects on skeletal cell membrane and increases insulin sensitivity and can increase adipocyte differentiation in cultured adipocytes [15-17]. Based on above- 
mentioned investigations, the present study was designed to evaluate the effects of Artemisia dracunculus L leaves aqueous extract on blood sugar, serum insulin trigleserid and liver enzymes in FDW male rats.

\section{Methods}

At the beginning of the experiment 48 Wistar-albino male rats, weighing 200-250g were selected from the Animal House of Zahedan University of Medical Sciences. The animals were kept in numbered cages. The rats had free access to water and food and were sustained in a room at 23 $\pm 2{ }^{\circ} \mathrm{C}$ temperature with a fixed 12: 12 -h artificial light/dark phase (lights on at 0600 hours by Timer Model: SUL180a, AC220V, China, 6 Am to $6 \mathrm{Pm}$ ), and humidity of 45-65\%.

After a week of accommodation, the rats were divided into control $(\mathrm{C})$, control Extract $(\mathrm{C}+\mathrm{E})$, fructose-Drinking water (FDW) and fructose-Drinking water and extract (FDW + E) groups randomly $(\mathrm{n}=12)$ as follow:

Group C received rodent's diet and water tap but did not receive any agents throughout the trial period.

Group C+E received water tap, rodent's diet and $100 \mathrm{mg} / \mathrm{kg}$ Artemisia dracunculus L leaves aqueous extract daily by gavages during the trial period.

Group FDW received fructose-enriched water $(10 \%, w / v)$ and rodent's diets at this time.

Group FDW + E received fructose-enriched water (10\%, $\mathrm{w} / \mathrm{v}$ ), rodent's diets and $100 \mathrm{mg} / \mathrm{kg}$ AD aqueous extract daily by gavages during the trial period.

\subsection{Preparation of The Extracts}

The plants were collected from a local area around Tehran, the capital of Iran in August, 2013 and identified by the biology taxonomy centre in science faculty of sistan and Baluchestan University, Zahedan, Iran. Artemisia dracunculus aerial was separated, shade-dried in a room temperature and then changed into powder by hand [18]. Extraction was obtained by mixing 20 grams of the powder in $200 \mathrm{~mL}$ of distilled water in a soxhelet apparatus for 24 hours. The prepared extract was filtered through a gauze cloth followed by filtration through a normal filter paper Watman no.1 $[12,18]$. The result was a brown aqueous extract which was dried subsequently in incubator for one day at $45^{\circ}$ and sustained in refrigerator temperature $\left(4^{\circ} \mathrm{C}\right)$.

At the end of the experiment ( 4 weeks) and after overnight fasting (12 - 14 hours) all animal were deeply anesthetized by diethyl ether (Merck Germany), decapitated and blood samples were collected without delay from cervical vessels. All blood samples were collected in normal vials and centrifuged at 3000rpm for 10 minutes to disconnect serum [19]. Serum was removed (BH-1200 type Iran) and accumulated at $-70^{\circ} \mathrm{C}$ for supplementary analyses. Serum insulin was measured by sensitive rat kit (DRG, instruments, GMBH, Germany), using double antibody Enzyme-linked immunosorbent assay (ELISA). Blood glucose, lipid profile and liver enzymes activity were measured blind by usual methods. Homa -IR scores were obtained with the following formula: Homa -IR = Fasting insulin $(\mu \mathrm{Iu}) \times$ Fasting plasma glucose $(\mathrm{mmol} / \mathrm{L}) / 22.5[19$, 20]. Serum AST and ALT, blood glucose, triglyceride (TG) and total cholesterol (TC) levels were measured using the standard methods adapted for RA 1000 Analyzer (Technicon, USA) using Pars Azmon kit Iran. Serum high density lipoprotein (HDL) was measured by precipitation of nonHDL lipoprotein with dextran/MgSO4, followed by enzymatic cholesterol assay. Low density lipoprotein (LDL) was calculated according to Friedewald formulas (Friedewald WT 1972).

These experimental animals were taken in accordance with recommendations from the declaration of Helsinki and internationally conventional standards for the employment of experimental animals, and they received institutional ethical authorization from the committee for animal research of Zahedan University of Medical Sciences (project No, 6000).

The normal distribution of data was approved by Kolmogorov-Smirnov test and then all data were analyzed by SPSS software v. 17; via one way ANOVA and Tukey tests. The results were expressed as mean \pm SD. Statistical differences were considered significant at $\mathrm{P}<0.05$.

\section{Results}

The results obtained from the present study indicated that insulin resistance indexes (IRI), in FDW groups (0.15 $\pm 0.06)$ were significant higher than that of $\mathrm{C}$ and $\mathrm{C}+\mathrm{E}$ groups (0.09 $\pm 0.03,0.13 \pm 0.06)$ but this parameter in FDW+E group (0.12 \pm 0.05$)$ significantly decreased compared to FDW group (Table 1). In addition, serum insulin, fasting blood sugar (FBS) and triglyceride in group FDW were significantly higher than those of $\mathrm{C}$ and $\mathrm{C}+\mathrm{E}$ groups, but these values in group FDW + E were significantly lower than group FDW (Table 1). Moreover, our findings revealed that Aspartate amino transferase (AST) and Alanine amino transferase (ALT) value in group FDW significantly increased in comparison to $\mathrm{C}$ and $\mathrm{C}+\mathrm{E}$ groups. On the other hands, AST and ALT values in group FDW + E significantly decreased compared to group FDW (Figures 1 and 2). In addition, Alkaline phosphatase (Alp) in all groups did not show any significant differences. 
Table 1. The Effects of Artemisia dracunculus Aqueous Extract on Blood Sugar, Serum Insulin, IRI and Lipid Profile in FDW Rats $(\mathrm{N}=12)$

\begin{tabular}{|c|c|c|c|c|c|}
\hline \multirow[t]{2}{*}{ Parameters } & \multicolumn{4}{|c|}{ Groups } & \multirow[t]{2}{*}{ PValue } \\
\hline & Group C & Group C + E & Group FDW & Group FDW + E & \\
\hline IRI & $0.09 \pm 0.03$ & $0.14 \pm 0.06$ & $0.15 \pm 0.05$ & $0.12 \pm 0.05^{*}$ & $\mathrm{P}<0.02$ \\
\hline Insulin, $\mu / \mathbf{L}$ & $0.24 \pm 0.15$ & $0.4 \pm 0.16$ & $0.49 \pm 0.14$ & $0.35 \pm 0.13^{*}$ & $\mathrm{P}<0.001$ \\
\hline FBS, mg/dL & $112.83 \pm 17.87$ & $108.75 \pm 10.73$ & $149.08 \pm 26.65$ & $121.08 \pm 13.77^{*}$ & $\mathrm{P}<0.004$ \\
\hline Triglyceride, mg/dL & $52.33 \pm 6.59$ & $59.33 \pm 17.52$ & $60.66 \pm 11.16$ & $48.18 \pm 6.21^{*}$ & $\mathrm{P}<0.05$ \\
\hline
\end{tabular}

"Asterisk show significant difference between groups $(\mathrm{P}<0.05)$. Mean $\pm \mathrm{SD}, \mathrm{n}=12, \mathrm{P}<0.05$

Table 2. The Effects of Artemisia dracunculus Aqueous Extract on Weight of Gain, Water and Food Intake in FDW Rats

\begin{tabular}{|c|c|c|c|c|c|}
\hline \multirow[t]{2}{*}{ Parameters } & \multicolumn{4}{|c|}{ Groups } & \multirow[t]{2}{*}{ P Value } \\
\hline & Group C & Group $\mathrm{C}+\mathrm{E}$ & Group FDW & Group FDW +E & \\
\hline Initial weight, gr & $202.50 \pm 12.5$ & $200.91 \pm 13.46$ & $200.41 \pm 15.19$ & $200.3 \pm 12.71$ & $P>0.05$ \\
\hline Final weight, gr & $236.8 \pm 7.3$ & $241.5 \pm 6.9$ & $249.7 \pm 8.5$ & $232.4 \pm 7.7^{*}$ & $\mathrm{P}<0.001, \mathrm{P}<0.05, \mathrm{P}<0.001$ \\
\hline Water intake, mL & $45.53 \pm 5.88$ & $48.30 \pm 3.19$ & $49.85 \pm 0.49$ & $49.56 \pm 2.66^{*}$ & $\mathrm{P}<0.02, \mathrm{P}<0.004$ \\
\hline
\end{tabular}

Asterisk show significant difference between groups $(\mathrm{P}<0.05)$. Mean $\pm \mathrm{SD}, \mathrm{n}=12, \mathrm{P}<0.05$

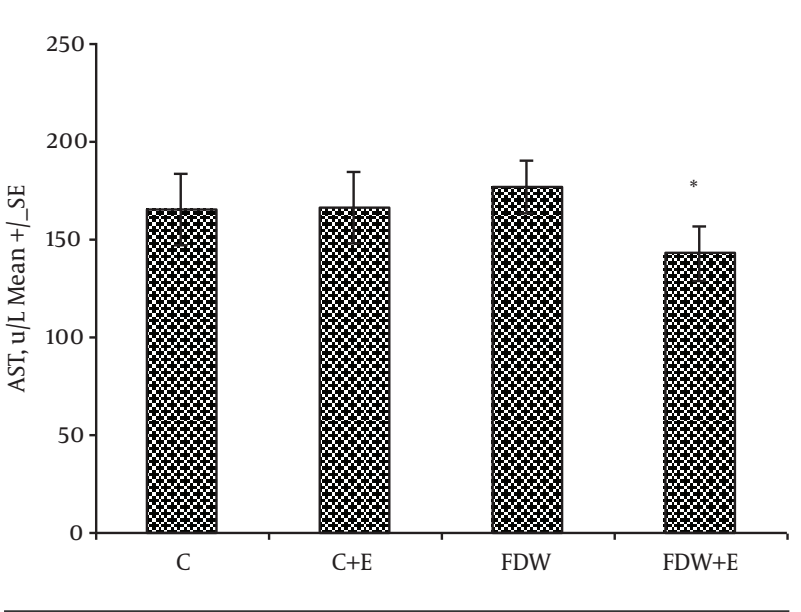

Figure 1. Comparison of AST (Miu) Activity Among the Control and Treated Male Rats. Asterisk Show Significant Difference Between Groups $N=12$, Values Represent Mean $\pm S D,{ }^{*}=P<0.05$

\section{Discussion}

Fructose utilization in Western diets has been increasing over the past 10 - 20 years and has been associated with an increase in obesity and metabolic disorders [21]. Fructose intake increases plasma triglycerides and changes hepatic glucose homeostasis [21]. Insulin and leptin are two important hormones which interfere in regulation of food intake and body weight gain in all vertebrata [21]. Di-

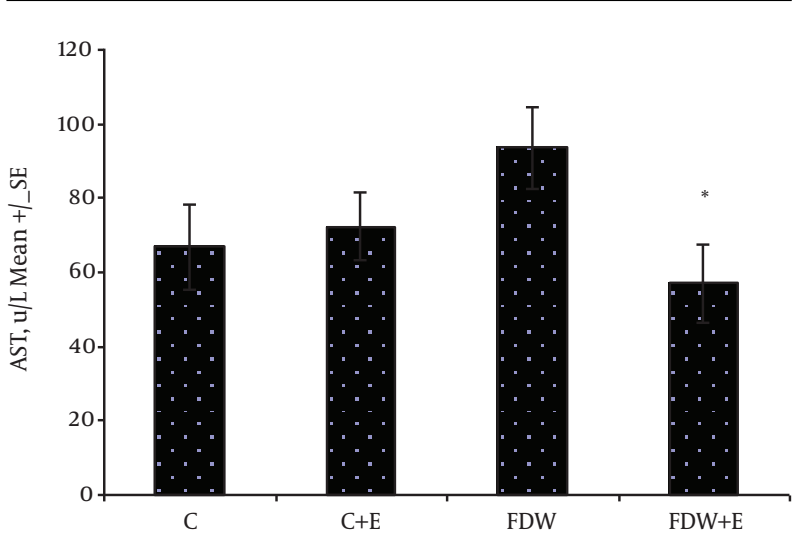

Figure 2. Comparison of ALT (Miu) Activity Among the Control and Treated Male Rats. Asterisk Show Significant Difference Between Groups $N=12$, Values Represent Mean $\pm S D,{ }^{*}=P<0.05$

etary fructose contributes to an increase in the input of energy, weight gain and alters blood lipid and carbohydrate homeostasis [22]. Insulin resistance and hyperinsulinemia are common in patients with metabolic syndrome, type II diabetes and essential hypertension, which are high risk factors of cardiovascular diseases, and play an important role in the development of coronary artery disease [5]. Our findings in the present study revealed that consumption of FDW (10\% weight/ volume) during a month in rat model, induced gain of weight, insulin resistance 
index (IRI), hyperinsulinemia and hyperglycemia in these animals. These results are supported by previous studies which indicated that fructose-fed and FDW cause increase obesity, insulin resistance, hyperinsulinemia and an abnormal insulin-signaling pathway in the central nervous system such as hippocampus which induced memory insufficiencies in animal model [23]. Literature indicates that in insulin resistance disorders such as type II diabetes and metabolic syndrome, insulin signaling is elevated which alters insulin action in skeletal muscles cell membranes and Artemisia dracunculus L. extract improved insulin action in skeletal muscles cell by increasing insulin signaling [23]. Investigational evidence revealed that metabolic pathways associated to glucose transport through cell membrane, glycolysis and cell signaling are probably influenced by Artemisia dracunculus L aqueous extract that possibly modulates carbohydrate metabolism as well as translocation of GLUT4 to the plasma membrane skeletal muscles cells [23]. This observation indicated that a possible molecular mechanism in the Artemisia dracunculus $\mathrm{L}$ aqueous extract components has enhanced glucose uptake, glucose transport in skeletal muscles and adiposcytes cell membrane to insulin and finally improved whole body insulin sensitivity [23]. Our findings in the present study showed that consumption of Artemisia aqueous extract improved IRI in FDW + E group compared to FDW group and the results are in agreement with literature. Moreover, some tests have shown that diabetic and insulinresistant patients have higher blood glucose and hyperinsulinemia compared to normal population [18]. The liver is a main organ for the protection of lipid, glucose and hormonal homeostasis. Fructose is a greatly lipogenic carbohydrate that has thoughtful metabolic effects on the liver inducing de nova lipogenesis and glucogenesis [24]. Investigation established that demonstration of the extracts from two Artemisia species in mice could enhance insulin-responsive by altering the serine phosphorelation in insulin receptor and affecting on monocyte chemotactic protein-1 levels in visceral white Adipocyte Tissue [25]. High fructose utilization in modern diet is frequently connected with insulin resistance, and distressed glucose homeostasis [26]. Choi MK et al. ( 2013) reported that the clinical uses of Artemisia capillaries is beneficial for hepatic disorders which is associated with alcohol and its mechanisms may involve both augmentation of antioxidant actions and modulation of proinflammatory cytokines [27]. Moreover, fructose rich diet cause increase of the hepatic triglyceride, total cholesterol levels, as well as serum activities of AST, ALT and lactate dehydrogenase [10]. Our results indicated that FDW increases serum AST and ALT in FDW group but these values decreased in FDW + E group. Our findings were agreement with Schaalan M et al. (2009) which showed that administration of FDW causes hyperglycemia and alters glucose homeostasis, lipid profile, insulin, and homeostasis model assessment (HOMA) index in westernized diet [28]. This alteration may be due to the high consumption of fructose in diet that possibly induced hepatocyte damage, leakage and liver enzymes increase in blood circulation.

In addition, Lim DW et al. (2013) reported that administration of Artemisia capillaris extract in high-fat diet (HFD) -induced obese rats could decrease the body gain of weight, serum triglyceride, total cholesterol and Lowe Density Lipoprotein in this model [29]. Moreover, in HFD rats which were treated with Artemisia capillaris extract, serum AST and ALT significantly decreased compared to HFD-control group [27]. Our findings also showed that FDW increased the liver injury which is associated with high AST and ALT but this value in group FDW+E were significantly lower than FDW group that was also supported with the findings of Lim DW et al. (2013).

\subsection{Conclusion}

Our finding in the present study indicated that Artemisia dracunculus leave aqueous extract has antihyperglycemic, anti-lipidemic and anti-hepatotoxicity effects in FDW rats.

\section{Acknowledgments}

This study was financially supported by the Deputy Research Center at Zahedan University of Medical Sciences (project No: 6000). We are grateful to Dr Ali Reza Nakhei for his kind cooperation.

\section{Footnotes}

Authors' Contribution: Mohammad reza Shahraki and Hamideh Mirshekari developed the original idea, protocol. Zahra samadi, Ahmmad reza shahraki and Elham shahraki, collected and analyzed data and wrote the manuscript.

Conflict of interest: The authors declare that they have no conflict of interest.

Funding/Support: Zahedan University of Medical Sciences.

\section{References}

1. Kovacevic S, Nestorov J, Matic G, Elakovic I. Dietary fructose-related adiposity and glucocorticoid receptor function in visceral adipose tissue of female rats. EurJ Nutr. 2014;53(6):1409-20. doi: 10.1007/s00394013-0644-1. [PubMed: 24420787]. 
2. Di Lorenzo C, Dell'agli M, Colombo E, Sangiovanni E, Restani P. Metabolic syndrome and inflammation: a critical review of in vitro and clinical approaches for benefit assessment of plant food supplements. Evid Based Complement Alternat Med. 2013;2013:782461. doi 10.1155/2013/782461. [PubMed: 23533519].

3. Pereira CD, Severo M, Araujo JR, Guimaraes JT, Pestana D, Santos A, et al. Relevance of a Hypersaline Sodium-Rich Naturally Sparkling Mineral Water to the Protection against Metabolic Syndrome Induction in Fructose-Fed Sprague-Dawley Rats: A Biochemical, Metabolic, and Redox Approach. Int J Endocrinol. 2014;2014:384583. doi: 10.1155/2014/384583. [PubMed: 24672546].

4. Guimaraes PS, Oliveira MF, Braga JF, Nadu AP, Schreihofer A, Santos RA, et al. Increasing angiotensin-(1-7) levels in the brain attenuates metabolic syndrome-related risks in fructose-fed rats. Hypertension. 2014;63(5):1078-85. doi: 10.1161/HYPERTENSIONAHA.113.01847. [PubMed: 24516106].

5. Fan CY, Wang MX, Ge CX, Wang X, Li JM, Kong LD. Betaine supplementation protects against high-fructose-induced renal injury in rats J Nutr Biochem. 2014;25(3):353-62. doi: 10.1016/j.jnutbio.2013.11.010. [PubMed: 24456735].

6. Rasool R, Ganai BA, Akbar S, Kamili AN. Free radical scavenging potential of in vitro raised and greenhouse acclimatized plants of Artemisia amygdalina. Chin J Nat Med. 2013;11(4):377-84. doi: 10.1016/S18755364(13)60055-2. [PubMed: 23845546].

7. Wu HW, Ren LF, Zhou X, Han DW. A high-fructose diet induces hippocampal insulin resistance and exacerbates memory deficits in male Sprague-Dawley rats. Nutr Neurosci. 2015;18(7):323-8. doi: 10.1179/1476830514Y.0000000133. [PubMed: 24856097].

8. Kheterpal I, Scherp P, Kelley L, Wang Z, Johnson W, Ribnicky D, et al. Bioactives from Artemisia dracunculus L. enhance insulin sensitivity via modulation of skeletal muscle protein phosphorylation. Nutrition. 2014;30(7-8 Suppl):S43-51. doi: 10.1016/j.nut.2014.05.001. [PubMed: 24985106].

9. Abolaji AO, Eteng MU, Ebong PE, Dar A, Farombi EO, Choudhary MI. Artemisia annua as a possible contraceptive agent: a clue from mammalian rat model. Nat Prod Res. 2014;28(24):2342-6. doi 10.1080/14786419.2014.936016. [PubMed: 25022560].

10. Choi MK, Han JM, Kim HG, Lee JS, Lee JS, Wang JH, et al. Aqueous extract of Artemisia capillaris exerts hepatoprotective action in alcoholpyrazole-fed rat model. J Ethnopharmacol. 2013;147(3):662-70. doi: 10.1016/j.jep.2013.03.065. [PubMed: 23548584].

11. Scherp P, Putluri N, LeBlanc GJ, Wang ZQ, Zhang XH, Yu Y, et al. Proteomic analysis reveals cellular pathways regulating carbohydrate metabolism that are modulated in primary human skeletal muscle culture due to treatment with bioactives from Artemisia dracunculus L.JProteomics. 2012;75(11):3199-210. doi:10.1016/j.jprot.2012.03.024. [PubMed: 22480907].

12. Lee HI, Seo KO, Yun KW, Kim MJ, Lee MK. Comparative study of the hepatoprotective efficacy of Artemisia iwayomogi and Artemisia capillaris on ethanol-administered mice. J Food Sci. 2011;76(9):T207-11. doi 10.1111/j.1750-3841.2011.02385.x. [PubMed: 22416729].

13. Craciunescu O, Constantin D, Gaspar A, Toma L, Utoiu E, Moldovan L. Evaluation of antioxidant and cytoprotective activities of Arnica montana L. and Artemisia absinthium L. ethanolic extracts. Chem Cent J. 2012;6(1):97. doi: 10.1186/1752-153X-6-97. [PubMed: 22958433].

14. Kim MH, Seo JY, Liu KH, Kim JS. Protective effect of Artemisia annua L. extract against galactose-induced oxidative stress in mice. PLoS One. 2014;9(7):e101486. doi: 10.1371/journal.pone.0101486. [PubMed: 24988450].

15. Obanda DN, Ribnicky DM, Raskin I, Cefalu WT. Bioactives of Artemisia dracunculus L. enhance insulin sensitivity by modulation of ceramide metabolism in rat skeletal muscle cells. Nutrition. 2014;30(7-8
Suppl):S59-66. doi: 10.1016/j.nut.2014.03.006. [PubMed: 24985108].

16. Richard AJ, Fuller S, Fedorcenco V, Beyl R, Burris TP, Mynatt R, et al. Artemisia scoparia enhances adipocyte development and endocrine function in vitro and enhances insulin action in vivo. PLoS One. 2014;9(6):e98897. doi: 10.1371/journal.pone.0098897. [PubMed: 24915004].

17. Anaya-Eugenio GD, Rivero-Cruz I, Rivera-Chavez J, Mata R. Hypoglycemic properties of some preparations and compounds from Artemisia ludoviciana Nutt.J Ethnopharmacol. 2014;155(1):416-25. doi: 10.1016/j.jep.2014.05.051. [PubMed: 24892833].

18. Honmore V, Kandhare A, Zanwar AA, Rojatkar S, Bodhankar S, Natu A. Artemisia pallens alleviates acetaminophen induced toxicity via modulation of endogenous biomarkers. Pharm Biol. 2015;53(4):571-81. doi: 10.3109/13880209.2014.934382. [PubMed: 25339313].

19. Kovacs D, Hegedus C, Kiss R, Sari R, Nemeth J, Szilvassy Z, et al. Mealinduced insulin sensitization is preserved after acute olanzapine administration in female Sprague-Dawley rats. Naunyn Schmiedebergs Arch Pharmacol. 2015;388(5):525-30. doi: 10.1007/s00210-015-1091-8. [PubMed: 25644372].

20. Kim TE, Lee DH, Kim YJ, Mok JO, Kim CH, Park JH, et al. The relationship between cognitive performance and insulin resistance in nondiabetic patients with mild cognitive impairment. Int J Geriatr Psychiatry. 2015;30(6):551-7. doi: 10.1002/gps.4181. [PubMed: 25060738].

21. Le KA, Tappy L. Metabolic effects of fructose. Curr Opin Clin Nutr Metab Care. 2006;9(4):469-75. doi: 10.1097/01.mco.0000232910.61612.4d. [PubMed: 16778579].

22. Bray GA, Nielsen SJ, Popkin BM. Consumption of high-fructose corn syrup in beverages may play a role in the epidemic of obesity. Am J Clin Nutr. 2004;79(4):537-43. [PubMed:15051594].

23. Velickovic N, Djordjevic A, Vasiljevic A, Bursac B, Milutinovic DV, Matic G. Tissue-specific regulation of inflammation by macrophage migration inhibitory factor and glucocorticoids in fructose-fed Wistar rats. BrJNutr. 2013;110(3):456-65. doi:10.1017/S0007114512005193. [PubMed: 23286672].

24. Dekker MJ, Su Q, Baker C, Rutledge AC, Adeli K. Fructose: a highly lipogenic nutrient implicated in insulin resistance, hepatic steatosis, and the metabolic syndrome. Am J Physiol Endocrinol Metab. 2010;299(5):E685-94. doi: 10.1152/ajpendo.00283.2010. [PubMed: 20823452].

25. Maham M, Moslemzadeh H, Jalilzadeh-Amin G. Antinociceptive effect of the essential oil of tarragon (Artemisia dracunculus). Pharm Biol. 2014;52(2):208-12. doi: 10.3109/13880209.2013.824007. [PubMed: 24074293].

26. Richard AJ, Burris TP, Sanchez-Infantes D, Wang Y, Ribnicky DM, Stephens JM. Artemisia extracts activate PPARgamma, promote adipogenesis, and enhance insulin sensitivity in adipose tissue of obese mice. Nutrition. 2014;30(7-8 Suppl):S31-6. doi: 10.1016/j.nut.2014.02.013. [PubMed: 24985103].

27. Habib M, Waheed I. Evaluation of anti-nociceptive, antiinflammatory and antipyretic activities of Artemisia scoparia hydromethanolic extract. J Ethnopharmacol. 2013;145(1):18-24. doi: 10.1016/j.jep.2012.10.022. [PubMed: 23117091].

28. Schaalan M, El-Abhar HS, Barakat M, El-Denshary ES. Westernizedlike-diet-fed rats: effect on glucose homeostasis, lipid profile, and adipocyte hormones and their modulation by rosiglitazone and glimepiride. J Diabetes Complications. 2009;23(3):199-208. doi: 10.1016/j.jdiacomp.2008.02.003. [PubMed: 18407527].

29. Lim DW, Kim YT, Jang YJ, Kim YE, Han D. Anti-obesity effect of Artemisia capillaris extracts in high-fat diet-induced obese rats. Molecules. 2013;18(8):9241-52. doi: 10.3390/molecules18089241. [PubMed: 23917113]. 